\title{
3D-printed hierarchical pillar array electrodes for high performance semi- artificial photosynthesis
}

Xiaolong Chen ${ }^{\mathrm{a}}$, Joshua M. Lawrence, ${ }^{\mathrm{c}}$ Laura Wey, ${ }^{\mathrm{c}}$ Lukas Schertel, ${ }^{\mathrm{a}}$ Qingshen Jing, ${ }^{\mathrm{b}}$ Silvia Vignolini, ${ }^{\mathrm{a}}$ Christopher Howe ${ }^{\mathrm{c}}$, Sohini Kar-Narayan ${ }^{\mathrm{b}}$, Jenny Z. Zhang ${ }^{\text {Ia }}$

${ }^{a}$ Department of Chemistry, University of Cambridge, Lensfield Road, Cambridge CB2 1EW, UK;

${ }^{\mathrm{b}}$ Department of Materials Science and Metallurgy, University of Cambridge, 27 Charles Babbage Road, Cambridge, CB3 OFS, UK;

${ }^{c}$ Department of Biochemistry, University of Cambridge, Tennis Court Road, Cambridge, CB2 1QW, UK;

${ }^{1}$ To whom correspondence may be addressed. Email: jz366@ cam.ac.uk.

\begin{abstract}
The re-wiring of photosynthetic bio-machineries to electrodes is a forward-looking semiartificial route for sustainable bio-electricity and fuel generation. Currently, it is unclear how the bio-material interface can be designed to meet the complex requirements for high biophotoelectrochemical performance. Here, we developed an aerosol jet printing method for generating hierarchical electrode structures using indium tin oxide nanoparticles. We printed libraries of micropillar array electrodes varying in height and sub-micron surface features and studied the energy/electron transfer processes across the bio-electrode interfaces. When wired to the cyanobacterium Synechocysis sp. PCC 6803, micropillar array electrodes with microbranches gave rise to substantially enhanced biocatalyst loading, light input, and electron flux output compared to state-of-the-art porous structures of the same height. Micropillar electrodes $600 \mu \mathrm{m}$ in height reached milestone mediated photocurrent densities of $245 \mu \mathrm{A} \mathrm{cm}{ }^{-2}$ (the closest thus far to theoretical predictions) and external quantum efficiencies of up to $29 \%$. This study provides a blueprint for bio-hybrid electrode design, and tools for where electrode architecture is vital for performance.
\end{abstract}




\section{Introduction}

Biocatalysts (from enzymes to living microorganisms) can be electrochemically connected (wired) to electrodes for biotechnological applications or fundamental studies ${ }^{1-4}$. In particular, cyanobacteria (photosynthetic bacteria) are free-living, self-repairing, abundant, solar-powered biocatalysts that can be wired to electrodes for electricity generation (biophotovoltaics, Fig. 1 a-b) and chemical synthesis ${ }^{2,5-7}$.

Presently, semi-artificial approaches for solar energy conversion are in the nascent phase, and photocurrent densities achievable by cyanobacterial electrodes have been calculated to be between $340 \mu \mathrm{A} \mathrm{cm} \mathrm{cm}^{-2}$ and $2400 \mu \mathrm{A} \mathrm{cm}^{-2}$ (at the lower estimate, the energy conversion efficiency already compares favourably to bio-fuel generation) ${ }^{8}$. However, typical photocurrents generated by cyanobacterial-electrodes are two orders of magnitude below this predicted value. This is despite substantial bioengineering efforts ${ }^{9,10}$ to increase biological electron output and the deployment of artificial electron mediators, which can be introduced to maximise electron harvesting from the photosynthetic electron transport chain (Fig. 1 b $)^{6,8}$. As such, the bottleneck likely lies with the electrode scaffold itself.

In photoelectrochemical reactions, the electrode structure must balance several factors (including photon, electron, substrate/product fluxes) to minimise reaction bottlenecks ${ }^{11}$. Where biocatalysts are employed, it must also compensate for the larger catalyst dimensions and lower overall loading ${ }^{2,6,12}$. The state-of-the-art electrode architecture in both protein-film and biofilm photoelectrochemistry is the hierarchically structured inverse opal (IO) porous electrode made from indium tin-oxide (ITO) $)^{7,13,14}$. The ITO material offers favourable conductivity, light scattering, and biocompatibility ${ }^{12}$; the macro-porosity offers a large electroactive surface area and aids biocatalyst penetration; and the meso substructure aids biocatalyst adhesion ${ }^{7,15}$. When the model cyanobacteria, Synechocystis sp. PCC 6803 (Synechocystis), were immobilised onto IO-ITO electrodes, a mediated photocurrent density of $14 \mu \mathrm{A} \mathrm{cm}^{-2}$ (Fig. 1c) and external quantum efficiency (EQE) of $2.7 \%$ were observed ${ }^{7}$. Further progress in electrode design has been limited by the lack of versatile fabrication techniques that can generate large libraries of hierarchical electrode structures.

Here, we developed an aerosol jet printing method for the efficient and reproducible fabrication of large libraries of hierarchical ITO electrodes where structural features spanning five orders of magnitude in length scales could be tuned. This enabled us to fabricate libraries of micropillar array electrodes (chosen for their favourable optical properties) ${ }^{16,17}$ using ITO 
nanoparticles (Fig. 1 d-f). We varied their heights and surface roughness to tune the electroactive surface areas in multiple dimensions and compared their properties against stateof-the-art IO-ITO electrodes. Using this approach, we expanded current understandings of electrode structure-activity relationships and arrived at a new generation of high-performing photosynthetic electrodes (Fig. 1c).

a)

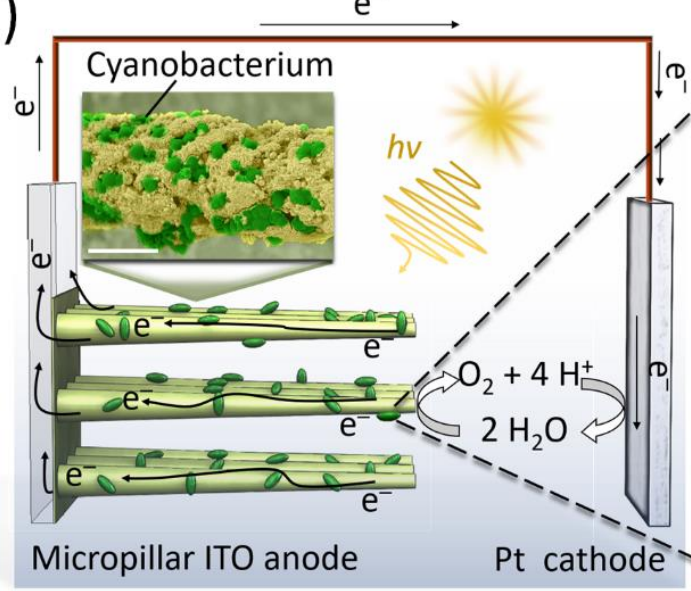

b)

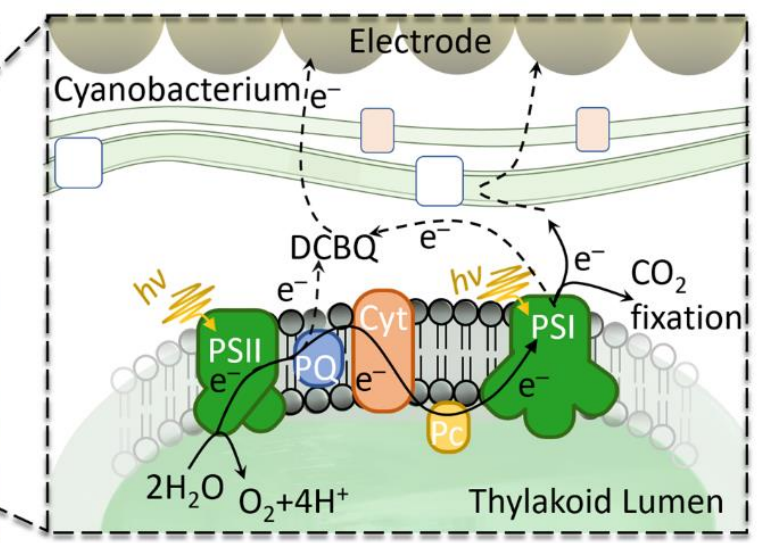

c)

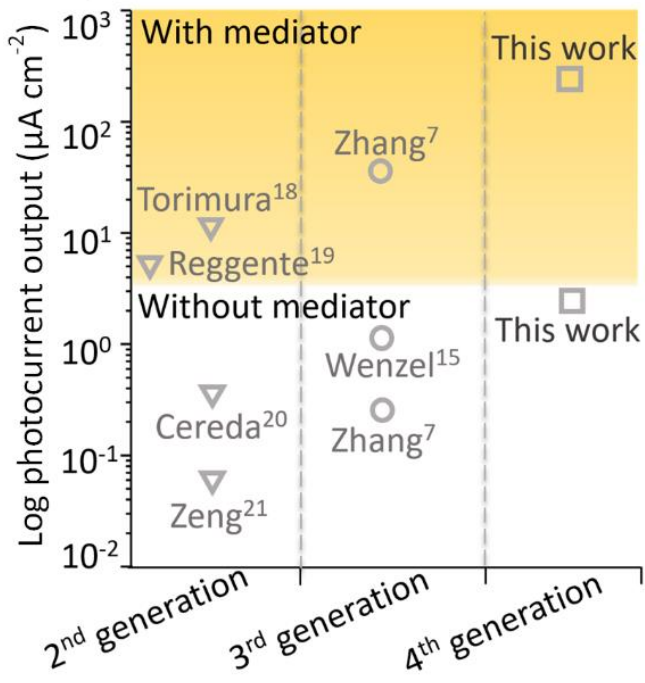

d) Aerosol jet printed micropillar library
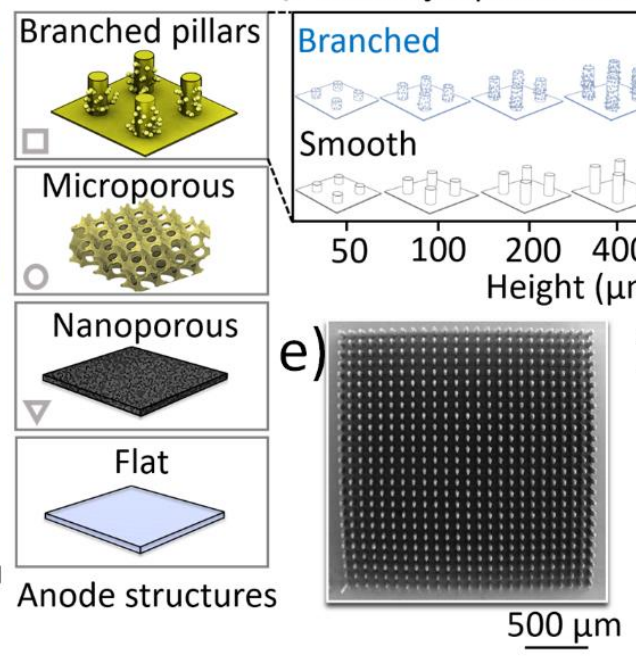

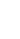

(1)

\section{.}


cyanobacterial-electrodes corresponding to different generations of electrode design. d) The library of aerosol jet printed micropillar ITO electrodes library presented in this study. e) SEM image of a printed micropillar array electrode (top view). f) SEM images of printed micropillar array electrodes (20 $\mu \mathrm{m}$ diameter, $100 \mu \mathrm{m}$ spacing) of different heights (side view).

\section{Aerosol jet printed ITO electrodes}

Aerosol jet printing is an additive manufacturing technique that can print metal nanoparticle inks with high design flexibility, resolution, and material deposition precision ${ }^{22,23-26}$ via a contactless direct write approach ${ }^{27-30}$. However, the fabrication of 3D hierarchical structures using this technique has not been reported. To establish an aerosol jet printing method for the fabrication of 3D structures using an ITO nanoparticle ink, significant deviations from classical ink compositions and printing parameters were needed.

In a typical procedure, a non-volatile ink precursor ${ }^{31,32}$ is aerosolized and entrained into a gas stream guided by an inert carrier gas such as $\mathrm{N}_{2}$ (Fig. 2a). Another focused coaxial stream of inert sheath gas collimates the aerosol stream at the deposition head to enable high precision aerosol deposition onto a substrate ${ }^{33}$. The ratio of the sheath gas to carrier gas flow rate and the nozzle size can be used to tune the cross-sectional profile and diameter of the printed area independent of the ink components (equation 1 in the SI) ${ }^{34}$. The solvent properties of the ink precursor determine the aerosol drop size and the rate of evaporation of the printed volume $\mathrm{s}^{35,36}$.

To produce pillar structures, an additional parameter - the gas focussing pressure ratio, which is the balance between the pressure of the carrier gas and the sheath gas (see equation 2 in SI), was tuned (Fig. 2b). It was observed that at ratios of between 2.4 and 3.5, pillars of up to 800 $\mu \mathrm{m}$ could be printed. Below this range, insufficient materials would be deposited; above this range, an 'overspill' effect occurred, resulting in a thick unstable pillar base (Fig. 2b inset).

We endowed the printed pillars with micro-scale roughness by changing to non-classical cosolvent systems, such as water (non-volatile) and methanol (volatile), in the ink precursor. The ratio of the water to methanol was varied to result in aerosol streams of different droplet sizes and volatilities (see Table S1); high water content $(>50 \% \mathrm{v} / \mathrm{v})$ gave rise to 'droplet-like' features (Fig. S1a); a methanol content of $50-65 \% \mathrm{v} / \mathrm{v}$ gave rise to 'smooth' nanoporous pillars (Fig. 2d, Fig. S1b); a methanol content of $65-75 \%$ v/v gave rise to sub-micron sized branched features (Fig. 2e); at a methanol content of $>75 \% \mathrm{v} / \mathrm{v}$, the diameter of the pillar became thin and brittle (Fig. S1c). The microbranches formed during the printing of the pillars was 

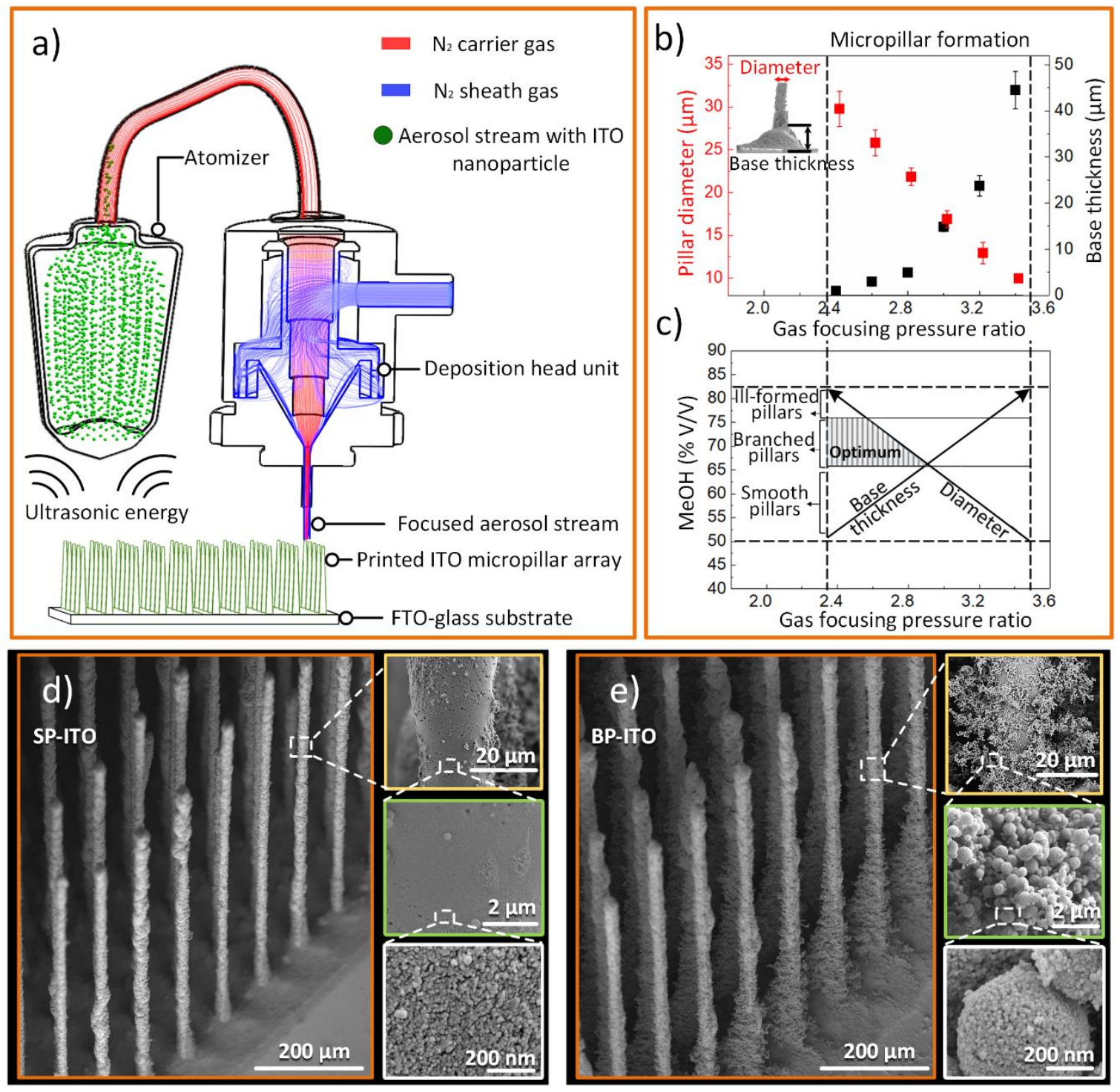

Figure 2: Aerosol jet printing of micropillar array electrodes. a) Schematic of the printing process. The ITO nanoparticle in methanol/water ink precursor was placed in an ultrasonic atomizer where it was aerosolized, entrained in a $\mathrm{N}_{2}$ carrier gas stream, and aerodynamically focused via a stream of coaxial $\mathrm{N}_{2}$ sheath gas. The micropillar ITO structures were printed onto a fluorine-doped tin oxide (FTO) coated glass. b) Printing parameters for producing micropillars: the gas focusing pressure ratio was identified as a key control parameter for tuning pillar shape. Inset: SEM image of a representative printed pillar with a base. c) Printing parameters for producing sub-micron roughness: an optimal operational window between gas focusing pressure ratio and ink $\mathrm{MeOH}$ content was identified to from micro-droplet branches. d) SEM image of a representative smooth micropillar ITO (SP-ITO) electrode with a height of $600 \mu \mathrm{m}$ and diameter of $20 \mu \mathrm{m}$. Images obtained at higher magnifications show nano-features resulting from the packing of the nanoparticles. The SPITO was fabricated using a focusing pressure ratio of 2.5 and $\mathrm{MeOH}$ volume fraction of $60 \%$. e) SEM image of a representative branched micropillar ITO (BP-ITO) electrode with the height of 600 $\mu \mathrm{m}$ and diameter of $20 \mu \mathrm{m}$. Images obtained at higher magnifications show micro-branch features comprising submicron droplets. Further magnification of the droplet features shows nano-features of the nanoparticles. The BP-ITO was fabricated using a gas focusing pressure ratio of 2.5 and $\mathrm{MeOH}$ volume fraction of $70 \%$. Error bars represent the standard error of the mean $(n=3)$. 
attributed to formation of smaller aerosol droplets at higher methanol contents. The small droplets near the sheath gas interface were too small to resist the turbulence at the side of the deposited area and were further broken up at the turbulent front. The submicron droplets formed follow wide trajectory patterns to result in the branched features with the volatility of the co-solvent mixture aiding rapid solidification of the structure.

We identified ideal conditions for generating arrays of well-formed 'smooth' micropillars (SP, Fig. 2 b) and hierarchically branched micropillars (BP, Fig. 2 c), and electrodes varying in pillar height $(50-800 \mu \mathrm{m})$ and roughness were printed for this study (Fig. 1D \& Fig. S2). The conditions used for the printing of the micropillar electrodes $(20 \mu \mathrm{m}$ diameter, $50-800 \mu \mathrm{m}$ height, $100 \mu \mathrm{m}$ centre-to-centre spacing) is documented in Table. $\mathbf{S 2}$.

\section{Characterization of the printed micro-pillar electrodes}

The printed micropillar electrodes were annealed and confirmed to retain the ITO composition via energy-dispersive X-ray spectroscopy (Fig. S3). Scanning electron microscopy (SEM) images of the libraries of the annealed SP-ITO and BP-ITO structures showed that the printed pillars within each array were uniform in height and diameter. We observed that the branching decreased with the height of the BP-ITO structure, with reduced branching at the top half of pillars at heights $>400 \mu \mathrm{m}$ (Fig. S2). This was attributed to the decrease in turbulence during the printing process as the distance between the printer nozzle and the tip of the pillar decreased.

The optical properties of the printed micropillar electrodes bare (in air) and adsorbed with Synechocystis (ca. $1.4 \mu \mathrm{m}$ in diameter, in aqueous medium) were measured with the aid of an integrating sphere (Fig. S4 - S6) and compared against benchmark third-generation IO-ITO electrodes (10 $\mu \mathrm{m}$ in pore sizes, $50 \mu \mathrm{m}$ in height, Fig. S7), shown in Fig. 3a-d. We observed that when irradiated with $0^{\circ}$ vertical incident white light $\left(1 \mathrm{~mW} \mathrm{~cm}^{-2}\right)$, the bare IO-ITO structure transmitted $4.4 \%$ and reflected $12.4 \%$ of the incident light; the SP-ITO array structure of the same height transmitted $32.5 \%$ and reflected $15.6 \%$ of the incident light, whereas the BP-ITO array structure transmitted $26.4 \%$ and reflected $20.2 \%$ of the incident light (Fig. 3c). As such, micropillar structures exhibited significantly enhanced light transmission and scattering compared to IO-ITO. From this, it could be predicted that much greater light harvesting by the Synechocystis-loaded electrodes is possible with the micropillar micro- 
branched structures. The remainder of the incident light could be attributed to light absorbance by the material or loss from in plane scattering. This was much higher for the IO-ITO structure (83.2\%) than for the SP-ITO (51.9\%) and BP-ITO structures (53.3\%) (Fig. S8). Light transmittance was previously reported to be poor through the porous IO-ITO electrode structures $^{37}$.

SP-ITO and BP-ITO electrodes ranging from $50 \mu \mathrm{m}$ to $800 \mu \mathrm{m}$ in height (such heights were not accessible for IO-ITO) were also characterised. The transmittance of the bare SP-ITO and BP-ITO electrodes linearly decreased with the increase in height, which was partially due to the corresponding increase in thickness of the pillar base. The overall transmittance of SP-ITO was higher than that of BP-ITO because the micro-branches blocked approximately $8 \%$ of the incident light; similarly, the reflectance of BP-ITO was enhanced due to the increased scattering arising from the branch-like feature ${ }^{38}$. The reflectance was proportional to the height of the bare pillar structures (Fig. 3c).

The electrodes were incubated with a concentrated culture of Synechocystis for $12 \mathrm{~h}$ in the dark, followed by gentle rinsing to remove loosely bound cells. The Synechocystis-loaded electrodes were immersed in aqueous BG11 medium with the transmittance and reflectance measured. Slight decreases in transmission and reflectance were observed for the Synechocystis-loaded IO-ITO electrodes (Fig. 3d), which corresponds to a very small change in absorbance $(<5 \%$; Fig. S8a). However, the transmittance and reflectance of the micropillar electrodes were significantly decreased in the presence of the adhered Synechocystis (Fig. 3d). The presence of cells on the electrodes increased light absorption by up to $40 \%$ for the tallest pillar structures (Fig. S8b). This could be attributed to light absorption by the chlorophyll $a(\mathrm{Chl} a)$ and other pigments ${ }^{39}$ which absorb light to drive photosynthesis.

The electroactive surface areas (EASAs) of the bare IO-ITO, SP-ITO, and BP-ITO electrode structures were measured using capacitance measurements (Fig. 3e and Fig. S9). When electrode structures of the same height $(50 \mu \mathrm{m})$ and geometric area $\left(0.0625 \mathrm{~cm}^{2}\right)$ were compared, IO-ITO had the highest EASA $\left(0.24 \mathrm{~cm}^{2}\right)$, followed by BP-ITO $\left(0.19 \mathrm{~cm}^{2}\right)$, then SP-ITO $\left(0.16 \mathrm{~cm}^{2}\right)$. The significant deviation of the measured EASA from the theoretical EASA (Fig. S9b) in the case of the IO-ITO is expected. Although regions of orderly IOstructures dominated, the electrode structure contains cracks (Fig. S7) and regions of heterogeneity which may be inaccessible to the electrolyte. In contrast, the theoretical EASAs of the micropillar electrodes matched almost exactly the experimentally measured values, 
which indicated that the electrolyte reached the entire printed structure and most of the ITO structure was electrochemically connected.

The cell loading capacity of each electrode structure was measured spectroscopically by assaying the $\mathrm{Chl} a$ content on the electrodes following experiments. As expected, the higher the pillar height of the micropillar electrodes, the higher the total Chl $a$ loading normalised by geometric area could be observed (Fig. S10); however, the relationship was not linear and Fig. 3f shows the Chl $a$ loading density normalised by the electrode EASA. The micropillar electrodes exhibited comparable normalised cell loadings to IO-ITO at pillar heights 50 and $100 \mu \mathrm{m}$, with the BP-ITO structure at pillar height of $200 \mu \mathrm{m}$ exhibiting the highest EASAnormalised cell density (3.69 $\mu \mathrm{g} \mathrm{cm}^{-2}$ ), which was $60 \%$ higher in absorbed Chl $a$ content compared to the IO-ITO electrode. The normalised $\mathrm{Chl} a$ content for the micropillar electrodes declined at heights above $200 \mu \mathrm{m}$; this could be attributed to the decrease in micro-branching in the case of the BP-ITO and gravitational effects (cells settling on the base) limiting cell attachment at the top of the pillars.

Confocal fluorescence microscopy was employed to acquire Z-stacks of the cell-loaded electrodes, exploiting the intrinsic fluorescence of Chl $a$ (Fig. 3g, and Fig. S11). Synechocystis cells were distributed non-uniformly throughout IO-ITO, with the greatest cell density being observed near the top of the electrode. Synechocystis cells were distributed uniformly along the pillars of the SP-ITO, with a greater density of cells depositing on the base of the pillars. Synechocystis cells were also distributed uniformly along the pillars of the BP-ITO, with a higher density of cells observed on the branches of the pillars. Enlarged colourised SEM images showed the distribution of cells from the top view of the electrodes (Fig. 3h). The cells were more densely distributed on the SP-ITO compared to IO-ITO, but most densely distributed on the BP-ITO, forming patches of biofilms. 
a)

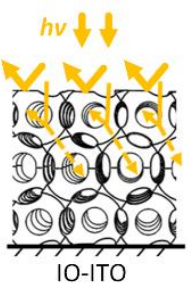

c)

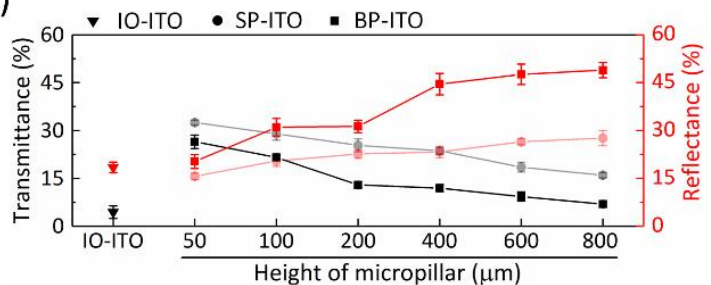

e)

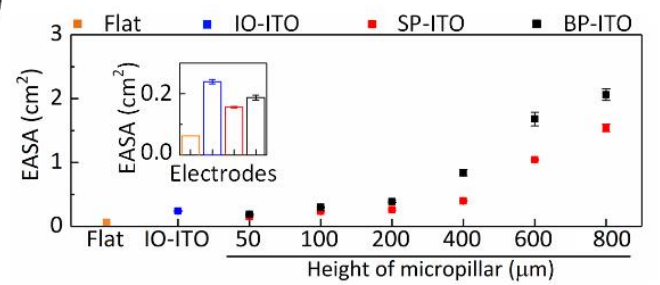

f)
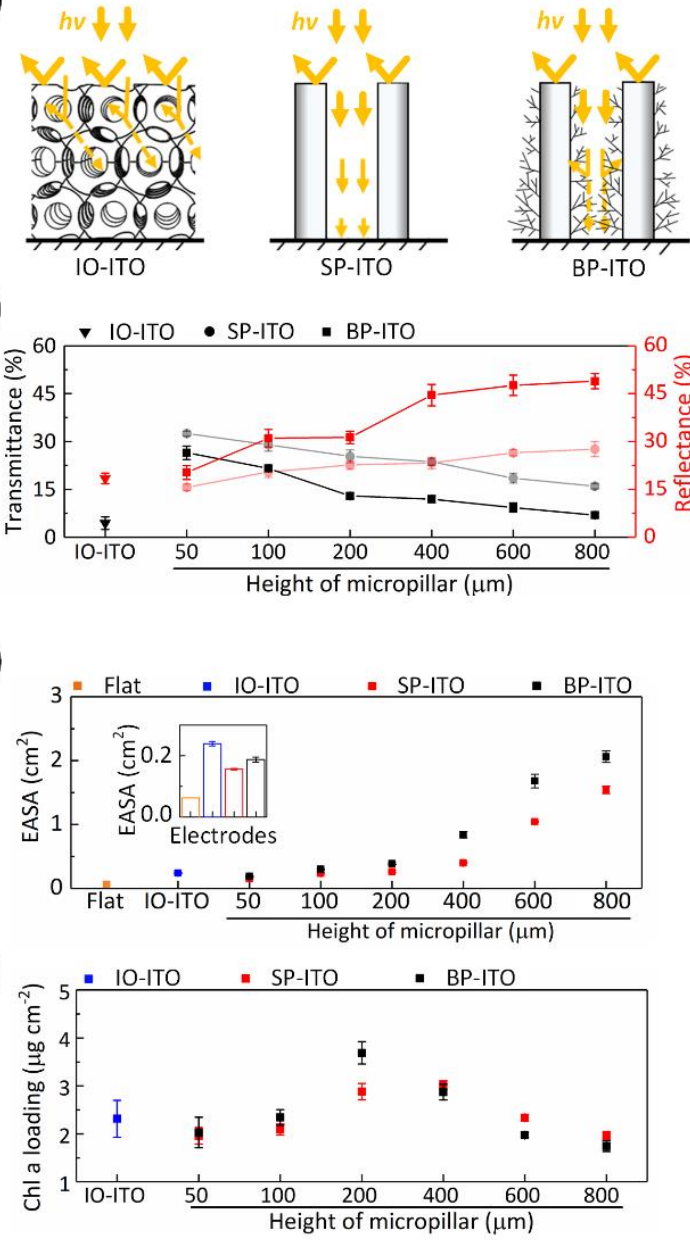

b)

d)
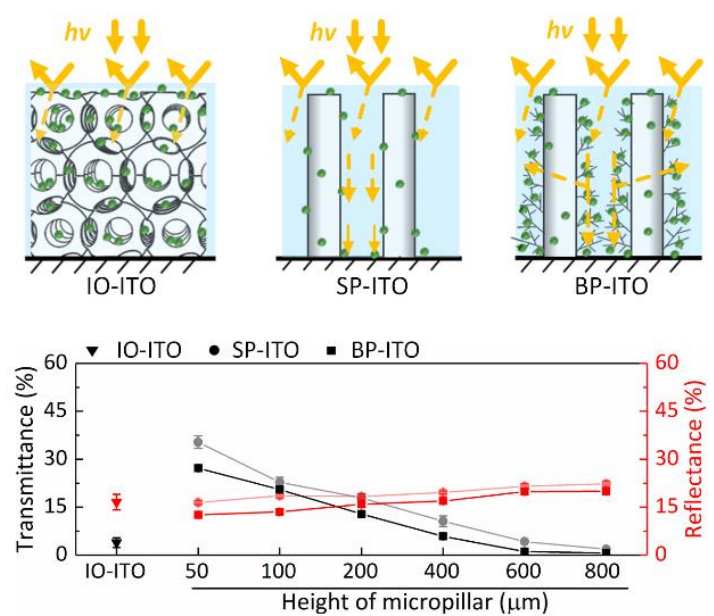

g)
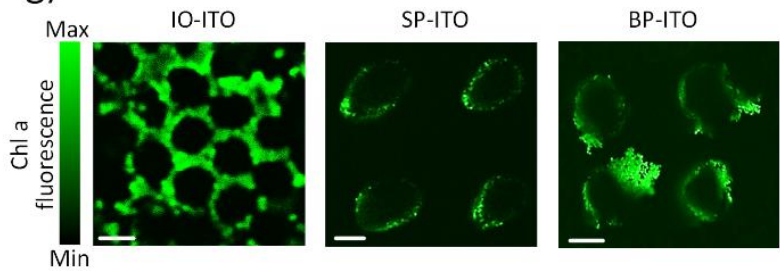

h)

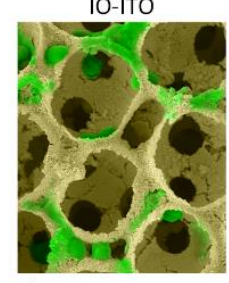

$5 \mu \mathrm{m}$
SP-ITO

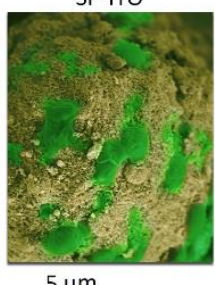

$5 \mu \mathrm{m}$
BP-ITO

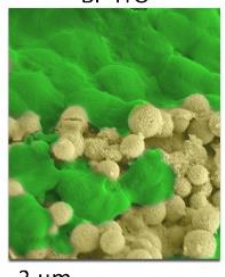

$2 \mu \mathrm{m}$

Figure 3: Printed micropillar electrodes exhibit high light transmission and cell loading. Light transmittance and reflectance studies of bare electrodes in air ( $\mathbf{a}$ and $\mathbf{c})$ and electrodes incubated with cells (b and d) using $0^{\circ}$ vertical incident white light $\left(1 \mathrm{~mW} \mathrm{~cm}{ }^{-2}\right)$. The IO-ITO electrodes were 50 $\mu \mathrm{m}$ in height, the SP-ITO and BP-ITO electrodes ranged from $50 \mu \mathrm{m}$ to $800 \mu \mathrm{m}$ in height. Concentrated suspensions of Synechocystis cells $\left(150 \mathrm{nmol}(\mathrm{Chl} a) \mathrm{mL}^{-1}\right.$ in BG11 medium) were incubated with electrodes for $12 \mathrm{~h}$, the electrodes were gently washed with the fresh medium before measurements. e) Electroactive surface area (EASA) of flat, IO-ITO, SP-ITO, and BP-ITO electrodes determined via capacitance measurements. Inset: zoomed in comparison of the EASA of flat, IOITO, SP-ITO, and BP-ITO electrodes all of $50 \mu \mathrm{m}$ height. f) EASA-normalised Chl $a$ loading on electrodes following $12 \mathrm{~h}$ incubation with Synechocystis and a gentle medium wash. g) Representative confocal microscopy images showing a cross-section through the middle of Synechocystis-loaded electrodes. Scale bar: $20 \mu \mathrm{m}$. h) Representative colourised SEM images of Synechocystis-loaded electrodes (top view). Error bars represent the standard error of the mean ( $\mathrm{n}=$ $3)$. 


\section{Bio-photoelectrochemical output}

The overall bio-photoelectrochemical output was assessed using chronoamperometry under dark/light cycles. A three-electrode configuration was employed with the Synechocystis-loaded electrode as the working electrode.

Under moderate light conditions $\left(\lambda_{680 \mathrm{~nm}}: 1 \mathrm{~mW} \mathrm{~cm}^{-2}\right)$ and in the absence of an exogenous electron mediator, Synechocystis-loaded micropillar ITO electrodes produced characteristic photocurrent profile of Synechocystis biofilms (Fig. 4a and Fig. S12) ${ }^{7}$. The photocurrent densities for Synechocystis-loaded SP-ITO and BP-ITO electrodes were higher than that of IOITO electrodes of the same height (Fig. $\mathbf{4 b}$ ), with photocurrent outputs of BP-ITO electrodes being twice those of IO-ITO. The addition of the commonly used membrane-permeable electron mediator 2,6-dichloro-1,4-benzoquinone (DCBQ) resulted in approximately 80, 100, and 110-fold increase for the IO-ITO, SP-ITO and BP-ITO electrodes (Fig. 4b), respectively, compared to their corresponding unmediated photocurrents. The effect of the mediator was greater for the micropillar array electrodes, which indicated that many cells on the electrode scaffold were not directly wired. This could be attributed to the multi-layer of cells on the base of the electrode or loosely attached cells on the periphery of the micro-branches.

The non-mediated photocurrent density was also assessed as a function of pillar height for the SP-ITO and BP-ITO electrodes (Fig. 4c and Fig. S13). The photocurrent density peaked at pillar heights of $600 \mu \mathrm{m}$ (SP: $1.5 \mu \mathrm{A} \mathrm{cm} \mathrm{cm}^{-2}$, BP: $1.93 \mu \mathrm{A} \mathrm{cm}$ ), with a drop observed for electrodes with $800 \mu \mathrm{m}$ pillars. The drop was attributed to the mechanical instability at 800 $\mu \mathrm{m}$, which may be the height limit of pillars printable by the aerosol jet printing method. The photocurrent output may be scaled up further via the height of the pillars, provided that the structural integrity issues can be resolved.

In this comparison study, Synechocystis-loaded BP-ITO electrodes at $600 \mu \mathrm{m}$ pillar height produced the highest non-mediated photocurrents. In the presence of mediators, EQE measurements carried out using red $(680 \mathrm{~nm})$ light at $1 \mathrm{~mW} \mathrm{~cm}{ }^{-2}$ for this electrode showed that $29 \%$ of the light was converted to current $\left(163 \mu \mathrm{A} \mathrm{cm} \mathrm{cm}^{-2}\right.$, Table S3), which aligned well with the $40 \%$ calculated light absorption by cells immobilised on this electrode (Fig. S8b). This is 10-fold higher in efficiency than the cells-loaded IO-ITO electrodes under analogous lighting conditions ${ }^{7}$.

To determine the maximum photocurrent output that could be generated using this electrode under more realistic conditions, we changed to a white light source and increased the intensity 
until maximum photocurrent output was observed at $3 \mathrm{~mW} \mathrm{~cm}$ c $^{-2}$ Under $3 \mathrm{~mW} \mathrm{~cm}^{-2}$ white light, non-mediated photocurrent outputs of $1.93 \mu \mathrm{A} \mathrm{cm}^{-2}$ and mediated photocurrents of 254 $\mu \mathrm{A} \mathrm{cm}^{-2}$ were observed (Fig. 4d and Table S3). This is an order of magnitude enhancement compared to previous benchmark photosynthetic biofilm systems and is within reach of the window of predicted theoretical photocurrent density that could be achieved by living photosynthetic electrodes (lower estimate: $340 \mu \mathrm{A} \mathrm{cm} \mathrm{cm}^{-2}$ ). Considering that less than $40 \%$ of the incident light was absorbed by the cells (Fig. S8), further improvements to the system are still possible via changes to the electrode structure to increase cell loading and light utilisation.
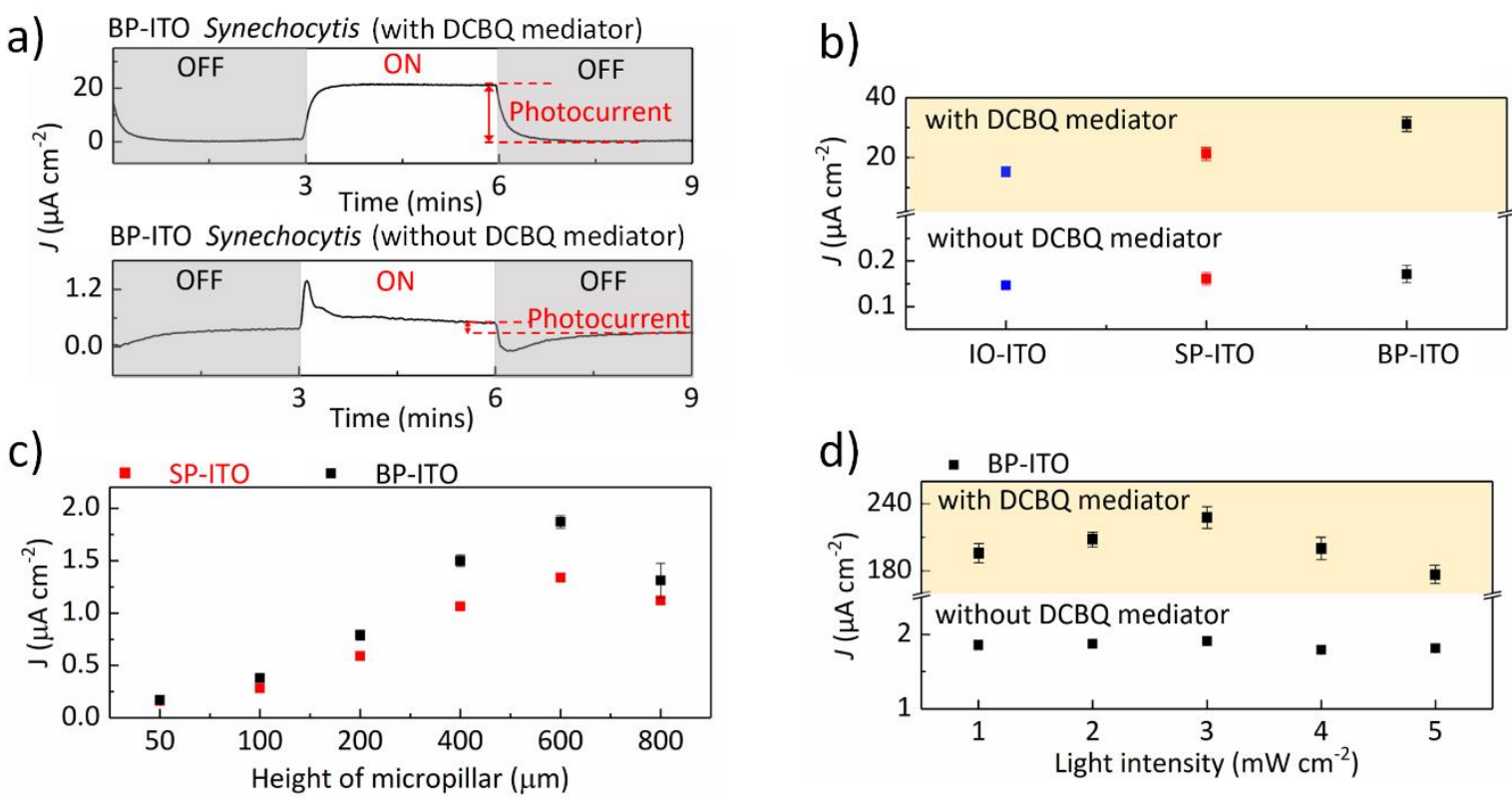

Figure 4: The photoelectrochemical performance of the Synechocystis-loaded electrodes. a) Representative photocurrent profile obtained from a Synechocystis-loaded BP-ITO electrode in presence and absence of the exogenous electron shuttle DCBQ $(1 \mathrm{mM})$. b) Summary of mediated and non-mediated photoelectrochemical performances of Synechocystis-loaded electrodes with different electrode architecture at equivalent thickness $(50 \mu \mathrm{m})$ under red light $\left(680 \mathrm{~nm}, 1 \mathrm{~mW} \mathrm{~cm}^{-}\right.$ ${ }^{2}$ ). c) Summary of non-mediated photoelectrochemical performances of the Synechocystis-loaded SPITO and BP-ITO electrodes with varying pillar heights without exogenous electron shuttles under red light $\left.(680 \mathrm{~nm}, 1 \mathrm{~mW} \mathrm{~cm})^{-2}\right)$. d) The photoelectrochemical performance of optimised Synechocystis-loaded BP-ITO electrodes (600 $\mu \mathrm{m}$ pillar height) under white light conditions of different intensities. Chronoamperometry experiments were performed with an applied potential of $0.3 \mathrm{~V}$ and $0.5 \mathrm{~V}$ vs SHE in the absence and presence of DCBQ, respectively, and in BG11 (pH 8.5) at $25^{\circ} \mathrm{C}$. Error bars represent the standard error of the mean $(n=3)$.

\section{Structure-activity relationships}

This study performed two sets of comparisons: (i) the performance between three different electrode architectures made with the same ITO nano-materials, height and geometric area; and 
(ii) the performance amongst libraries of micropillar ITO electrodes of different heights and surface roughness.

In the first comparison (Fig. 5a and Fig. S12), Synechocystis-loaded SP-ITO electrodes (50 $\mu \mathrm{m}$ height) yielded 2-fold higher mediated photocurrent densities than the Synechocystisloaded IO-ITO electrode despite the macro-porous structure exhibiting greater electron efflux capacity (21\% higher EASA and $13 \%$ higher cell loading). This infers that light flux into the bio-photoelectrode interface was limiting performance of the IO-ITO structure. Synechocystisloaded BP-ITO electrodes resulted in mediated photocurrent densities of $34 \mu \mathrm{A} \mathrm{cm}{ }^{-2}$, which was higher than that of the SP-ITO electrodes $\left(25 \mu \mathrm{A} \mathrm{cm}^{-2}\right)$; this was attributed to the microbranching resulting in enhancements to both electrode light reflection (> 30\%) and EASA (> $15 \%)$.

In the second comparison, a Spearman's rank correlation matrix was calculated from the library of micropillar structures to identify correlations between the properties of these electrodes (Fig. 5b and Fig. S14). Significant and strong correlation was observed between the pillar height of electrodes and their EASA $\left(\mathrm{R}=0.96, p=6.5 \times 10^{-7}\right)$, transmittance $\left(\mathrm{R}=-0.79, p=2.16 \times 10^{-}\right.$ $\left.{ }^{3}\right)$, reflectance $\left(\mathrm{R}=0.71, p=1.01 \times 10^{-2}\right)$, Chl $a$ loading $\left(\mathrm{R}=0.96, p=6.5 \times 10^{-7}\right)$ and photocurrent density $\left(\mathrm{R}=0.86, p=3.09 \times 10^{-4}\right)$. This infers that the larger surface of taller pillars provides more sites for cell loading and also improves the trapping of light within the structure. No significant correlation was observed between pillar micro-roughness and the photocurrent density across the library, though significant differences were observed for pillars of certain heights. This suggests that the macroscale features of micropillar electrodes (in this instance, pillar height) are the most important consideration for boosting photocurrent output. However, significant correlations were observed between micro-roughness and reflectance $(\mathrm{R}$ $=0.63, p=2.89 \times 10^{-2}$ ); this suggests that micro-roughness is important for introducing favourable photonic effects (important for enhancing performance in low light conditions). 
a)

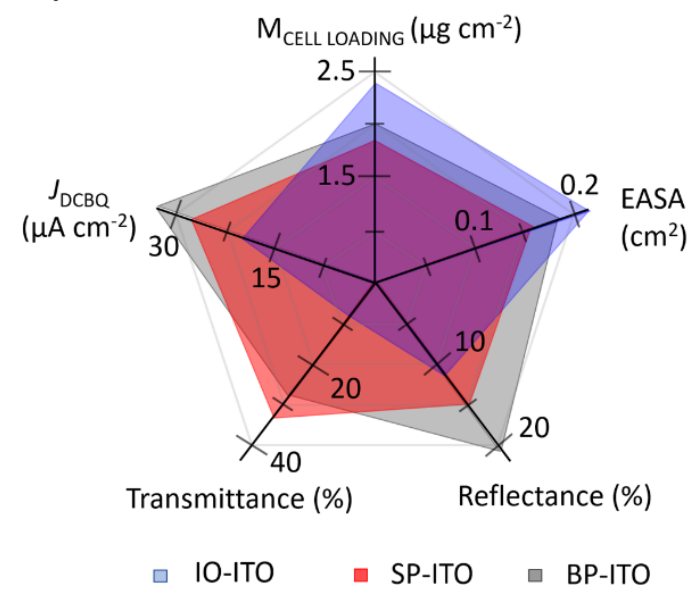

b)

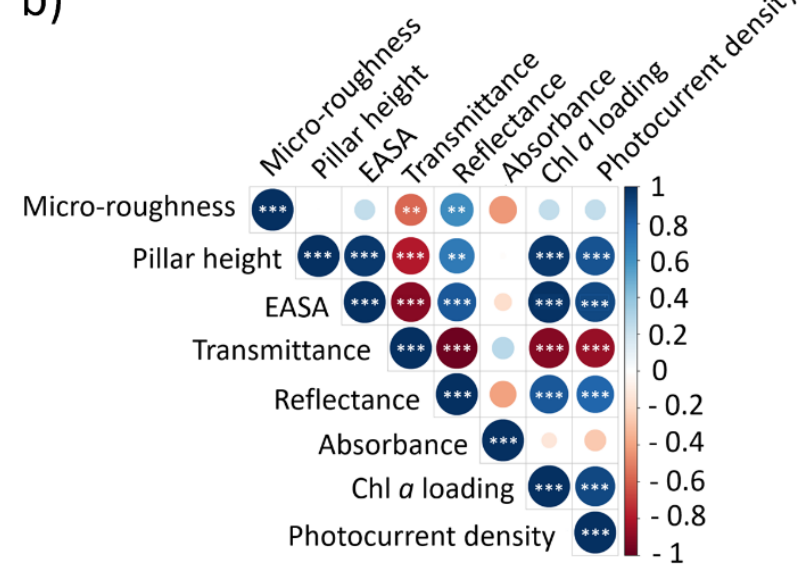

Figure 5: Structure-activity relationship analysis: a) Radar chart comparison between IO-ITO, SPITO and BP-ITO electrode structures $(50 \mu \mathrm{m}$ in height). b) Spearman's rank correlation matrix of micropillar electrodes and their properties. The scale bar represents the strength and sign of the correlation coefficient $(\mathrm{R})$, with blue indicating a positive and red a negative correlation coefficient. Stars indicate significance where $*$ is $p \leq 0.1, * *$ is $p \leq 0.05$ and $* * *$ is $p \leq 0.01$.

\section{Conclusion}

We established a 3D printing methodology for generating micropillar array structures with multi-scalar hierarchical features. This methodology can be applied to fields where electrode structural considerations are important for performance, particularly as we demonstrate that multi-dimensional features ranging the hundred-micron, submicron and nano-scales can be tuned.

Our fabrication method enabled rare comparison studies to be performed over a large library of electrodes to identify the bottlenecks currently limiting bio-photoelectrodes. We reveal that although the previous generation of macroporous electrodes offered large EASA for high biocatalyst loading, light and electrolyte penetration was limited through the structure. The micropillar electrode design overcame this issue, and the addition of submicron-roughness to the surfaces boosted EASA, cell loadings and light harvesting. Applying the lessons from this study, future electrode design within the field of photoelectrochemistry should prioritise light flux considerations before introducing highly structured architectures to boost electron flux capacity. 
The photocurrent outputs (up to $245 \mu \mathrm{A} \mathrm{cm}^{-2}$ for mediated electron transfer and up to $1.93 \mu \mathrm{A}$ $\mathrm{cm}^{-2}$ for direct electron transfer) demonstrated here is a milestone in bio-photoelectrochemistry. It brings us one order of magnitude closer to the photocurrents needed for living photosynthetic cells to be competitive in bioenergy generation. For example, when this bio-anode is paired with an appropriate cathode to result in a typical voltage difference $(0.213 \mathrm{~V})$, power densities of $541 \mathrm{~mW} \mathrm{~m}^{-2}$ can be estimated - this compares already favourably against biofuel generation $\left(<403 \mathrm{~mW} \mathrm{~m}^{-2}\right)^{8}$. Lessons from this study will guide the development of future highperforming bio-electrode structures; however, advancements to electron mediation strategies (to replace DCBQ) will be important for long-term high performance ${ }^{40}$. This study demonstrates the power of 3D printing in 3D electrode design and opens new directions for understanding and enhancing the bio-electrode interface.

\section{Methods}

\section{Electrode fabrication}

IO-ITO electrodes: The microporous electrodes $(10 \mu \mathrm{m}$ pores, $\sim 3 \mu \mathrm{m}$ channels, $50 \mu \mathrm{m}$ thickness) were prepared using templated synthesis via an infiltration approach adopted from a previous study ${ }^{1}$, with small adjustments. Briefly, cleaned FTO-coated glass (sheet resistance in $8 \Omega \mathrm{cm}^{-2}$, Sigma Aldrich) were applied with a mould in the form of a hole with geometric surface area $0.0625 \mathrm{~cm}^{2}$ (prepared from electrical tape $25 \mathrm{~mm}$ thick). The FTO-coated glass with the attached mould was $\mathrm{O}_{3}$ treated (UV/Ozone Procleaner, Bioforce Nanosciences) for 15 min. Polystyrene beads (10 $\mu \mathrm{m}$ diameter, $2.54 \% \mathrm{w} / \mathrm{v}$ suspension in water, Alfar Asaer) were spun down via centrifugation (12300 g, $5 \mathrm{~min}$ ). The polystyrene bead sediments were resuspended with supernatant using $1 / 4$ of the original supernatant. Aliquots of the polystyrene bead suspension $(30 \mu \mathrm{L})$ were spread inside the mould of the FTO-coated slides. To allow for uniform sedimentation, the polystyrene beads were slowly dried by evaporation over $3 \mathrm{~h}$, followed by 10 mins sintering at $80{ }^{\circ} \mathrm{C}$. ITO nanoparticles $(80 \mathrm{mg},<50 \mathrm{~nm}$ diameter, Sigma Aldrich) were combined with a mixture of $\mathrm{MeOH}(252 \mu \mathrm{L})$ and deionized water $(48 \mu \mathrm{L})$. The ITO suspension was ultrasonicated for $5 \mathrm{~h}$ at $50^{\circ} \mathrm{C}$. The ITO suspension $(6.8 \mu \mathrm{L})$ was dropcast onto the polystyrene bead template and allowed to infiltrate slowly over approx. $1 \mathrm{~h}$. The electrodes were annealed at $500{ }^{\circ} \mathrm{C}$ for $20 \mathrm{mins}$ (ramp rate: $1{ }^{\circ} \mathrm{C} \mathrm{min}^{-1}$, carbolite furnace). 
SP-ITO and BP-ITO electrodes: All micropillar structures were fabricated with an aerosol jet printer (AJ200, Optomec Inc.) using an ITO precursor (36\% w/v) prepared from a solvent mixture of water and $\mathrm{MeOH}$. The ratio of water to $\mathrm{MeOH}$ was systematically changed from 6:1 to 1:6 to investigate the effect of solvent on pillar geometry formation. This ratio range is compatible with the ultrasonic atomizer with precursor viscosity no higher than $5 \mathrm{cP}$. The ITO ink precursor $(2 \mathrm{~mL})$ was ultrasonicated at $50{ }^{\circ} \mathrm{C}$ for $3 \mathrm{~h}$ prior to use. The ITO precursor was then transferred to the ultrasonic atomizer, which was maintained at a constant temperature of $14{ }^{\circ} \mathrm{C}$ using a water jacket.

The aerosol jet printer was situated inside a glovebox (MBRAUN) in an ultra-clean and highly purified gas environment with $\mathrm{O}_{2}$ and $\mathrm{H}_{2} \mathrm{O}$ concentrations $<1$ ppm. Dry $\mathrm{N}_{2}$ (HPCL grade, 99.998\%) was used as the carrier gas of the ITO stream, which was set between 0-50 standard cubic centimetres minute ( $\mathrm{sccm}$ ). The focused sheath gas was set between 0-200 sccm, and the atomized current was maintained at $0.4 \mathrm{~mA}$. The flowrates of the gasses were measured at their inlets of the printer. A $7.5 \mathrm{~mm}$ long nozzle with a diameter of $150 \mu \mathrm{m}$ was used for printing.

More information on the operational mechanisms of the aerosol jet spray printing process can be found in the SI.

\section{Electrode characterisation}

SEM and EDX: SEM was conducted with a Tescan Mira3 FEG-SEM operated at an acceleration voltage of $5 \mathrm{kV}$. All the imaged samples were sputtered with a $10 \mathrm{~nm}$ layer of $\mathrm{Pt}$ to prevent charging. EDX was performed on Zeiss Auriga using $20 \mathrm{kV}$ electron acceleration voltage, at a working distance of $5 \mathrm{~mm}$.

Transmission and reflection measurements: The electrode structures (IO-ITO, SP-ITO and BP-ITO) were sealed by a 3D-printed circular silicon mould (diameter: $1 \mathrm{~cm}$ ), covered by thin glass (thickness: $0.13 \mathrm{~mm}$, Sigma Aldrich). A light source (Xenon lamp Ocean Optics HPX2000) was coupled in an optical fibre (Thorlabs FC-UV100-2-SR) to illuminate the sample placed in front (transmission) or at the back (reflection) of an integrated sphere (Labsphere). The scattered light was collected into an optical fibre which was connected to a spectrometer (Avantes HS2048) for the measurements (Fig. S4). For the transmission measurements, the illuminated light signal was normalized to the intensity (equivalent to $1 \mathrm{~mW} \mathrm{~cm}{ }^{-2}$ at wavelength $680 \mathrm{~nm}$ ) without samples. For the reflection measurements, the light signal was normalized according to the coating of the integrated sphere. FTO-glass (thickness: $3 \mathrm{~mm}$, 
Sigma Aldrich) and cover-slip glass (thickness: $0.13 \mathrm{~mm}$, Sigma Aldrich) were measured as the reference for both reflection and transmission tests. An unpolarized light was used with integration time of $2 \mathrm{~s}$.

Electroactive surface area characterisation: The EASA was calculated from the capacitance of the electrodes, which was measure using cyclic voltammetry are different scan rates using the ITO electrodes as the working electrode, Pt mesh as the counter electrode and $\mathrm{Ag} / \mathrm{AgCl}$ (saturated $\mathrm{KCl}$ ) as the reference electrode. The electrolyte used was 1,1'-ferrocenedimethanol (1 mM in 1-butyl-3-methylimidazolium hexafluorophosphate). The capacitance was analysed using the equation (1) below:

$$
\frac{i_{a}-i_{c}}{2}=C v
$$

Where $C$ is the capacitance $(\mathrm{F}), i_{a}$ is the anodic current (A) measured at $0.1 \mathrm{~V} v s . \mathrm{Ag} / \mathrm{AgCl}$ (saturated $\mathrm{KCl}$ ), $i_{c}$ is the equivalent cathodic current (A) measured at $0.1 \mathrm{~V} v s . \mathrm{Ag} / \mathrm{AgCl}$ (saturated $\mathrm{KCl}$ ) and $v$ is scan rate $\left(\mathrm{V} \mathrm{s}^{-1}\right)$. An aerosol jet sprayed flat ITO surface $\left(0.0625 \mathrm{~cm}^{2}\right)$ was used as the planar surface reference.

\section{Cell-loaded electrodes characterisation}

Cell culturing: Synechocystis sp. strain PCC 6803 (Howe lab wild type strain) ${ }^{41}$ was grown photoautotrophically at $30{ }^{\circ} \mathrm{C}$ with a continuous white light intensity of $50 \mathrm{uE} \mathrm{m}^{-2} \mathrm{~s}^{-1}$ in BG11 medium supplemented with $10 \mathrm{mM} \mathrm{NaHCO}_{3}{ }^{42}$. Glycerol stocks were streaked onto agar plates (with solid medium containing $1.5 \%$ agar). Individual colonies were picked to inoculate liquid cultures, which were air-bubbled and shaken at $120 \mathrm{rpm}$. Growth of liquid cultures was monitored by attenuance ( $\left.\mathrm{OD}_{750}\right)$ with $\mathrm{Chl} a$ concentration determined by absorbances at 680 and $750 \mathrm{~nm}:\left(\mathrm{A}_{680}-\mathrm{A}_{750}\right) \times 10.814^{43}$.

The early stationary phase planktonic cultures $\left(\mathrm{OD}_{750}=1\right)$ were centrifuged for $10 \mathrm{~min}$ at 5,000 $\mathrm{g}$ and resuspended in fresh BG11 to a concentration of $150 \mathrm{nmol} \mathrm{Chl} a \mathrm{~mL}^{-1}$ before photoelectrochemitry experiments.

Cell-loading on electrodes: A 3D-printed silicon circular mould ( $\left.\operatorname{area}=0.75 \mathrm{~cm}^{2}\right)$ with height of $3 \mathrm{~cm}$ was used to surround the printed electrode structure on the FTO coated glass in a watertight manner. Concentrated Synechocystis suspensions $\left(30 \mu \mathrm{l}, 150 \mathrm{nmol} \mathrm{Chl} a \mathrm{~mL}^{-1}\right)$ were pipetted into the silicon mould and allowed to incubate in the dark for $12 \mathrm{~h}$. The mould functioned to decrease the rate of cell settling to the bottom of the electrodes. After $12 \mathrm{~h}$, the 
cell loaded electrodes were carefully submerged with fresh BG11 to wash off loosely bound cells.

The cell loadings on the electrodes were determined using a previously reported method ${ }^{7}$. The concentration of Chl $a$ loaded onto electrodes were typically measured immediately after photoelectrochemical or light transmission/reflection experiments. The cell-loaded ITO electrodes were scraped with a pipette tip from the substrate glass and into a microcentrifuge tube containing $500 \mu \mathrm{L} \mathrm{MeOH}$. To avoid photodegradation of $\mathrm{Chl} a$, the vial was covered with aluminium foil, then placed in an ultrasonic bath cooled to $0^{\circ} \mathrm{C}$ and sonicated for $30 \mathrm{~min}$ to extract all $\mathrm{Chl} a$. The suspension was further centrifuged at $13,000 \mathrm{~g}$ for $3 \mathrm{~min}$ and the supernatant was analysed by UV-Vis spectrometry using the absorbance $665 \mathrm{~nm}$ and $750 \mathrm{~nm}$ : $\left(\frac{A_{665}-A_{750}}{79.95(\text { Chl a mg })^{-1} \mathrm{~mL} \mathrm{~cm}^{-1}}\right)$

All UV-visible spectrophotometry measurements were made on a Varian Cary 50 Bio.

Confocal fluorescence microscopy: A Leica TCS SP8 confocal microscope with a 10x magnification objective was used to image cell attachment to the electrode structures following cell loading. A $633 \mathrm{~nm}$ laser $(\mathrm{HeNe})$ was employed to excite biological cells, and the fluorescence was collected by a low-noise hybrid detector in the range of 650-750 nm. Z-stack images were acquired via sequential scanning downward from electrode surface to FTO glass substrate to the depth of $50 \mu \mathrm{m}$ (Z-depth $0.5 \mu \mathrm{m})$.

Photoelectrochemistry: Photoelectrochemical experiments were carried out using an Ivium CompactStat potentiostat. A Pt mesh counter electrode and an $\mathrm{Ag} / \mathrm{AgCl}$ (saturated $\mathrm{KCl}$ ) reference electrode was used in combination with the cell-loaded ITO working electrodes. The water-jacketed electrochemical cell was maintained at $25{ }^{\circ} \mathrm{C}$ and BG11 medium (pH 8.5) was used as electrolyte. A red LED light source $(\lambda=680 \mathrm{~nm}$, THORLABS $)$ at intensity of $1 \mathrm{~mW}$ $\mathrm{cm}^{-2}$ (which is equivalent to $56.8 \mu \mathrm{mol}$ photons $\mathrm{m}^{-2} \mathrm{~s}^{-1}$ ) was employed in the comparison studies. Chronoamperometry was carried out under light/dark cycles of $300 \mathrm{~s}$ on and $300 \mathrm{~s}$ off with an applied potential of $0.1 \mathrm{~V}$ (vs. $\mathrm{Ag} / \mathrm{AgCl}$ ) used in the absence of an exogenous electron mediator, and $0.3 \mathrm{~V}$ (vs. $\mathrm{Ag} / \mathrm{AgCl})$ used in the presence of DCBQ $(1 \mathrm{mM})$. Where stated, a white LED light source (THORLABS, SM2F32-A) was also employed, and light-responses were tested at intensities that varied from 1 to $5 \mathrm{~mW} \mathrm{~cm}^{-2}$. All chronoamperometry experiments were given $90 \mathrm{~s}$ of equilibrium period in the dark prior to initial light irradiation. It should be noted that in the presence of DCBQ, the mediated photocurrents drop rapidly, possibly due to 
diffusional limitations of the mediator in the unstirred electrolyte and partial cytotoxicity effects $^{40,41}$. As such, only the initial (first light cycle) mediated photocurrents were analysed.

EQE Calculations: EQE is calculated based on percentage of incoming photons converted to electrons at a certain bias potential and can be calculated by equation listed below:

$$
\mathrm{EQE}(\%)=\frac{123.98 J\left(\mu \mathrm{A} / \mathrm{cm}^{2}\right)}{[\lambda(\mathrm{nm})]\left[I\left(\mathrm{~mW} / \mathrm{cm}^{2}\right)\right.}
$$

Statistical analysis: Statistical analysis was performed in the R environment (v4.0.4) $)^{44}$. Quantitative data of electrodes were expressed as mean $(n=12)$. Micro-roughness was expressed as a binary integer $(0=$ pillars-ITO, $1=$ BP-ITO $)$. Pairwise Spearman's rank correlations and $\mathrm{p}$-values were calculated and visualised as a correlation matrix using the 'corrplot' package ${ }^{45}$.

\section{Acknowledgements}

This work was supported by the Biotechnology and Biological Sciences Research Council (BB/M011194/1 to JML, BB/R011923/1 to JZ, and XC), the Cambridge Trust (LTW), the Isaac Newton Trust (SCHERTEL SNSF3 to LS). S.K.-N. is grateful for support from a European Research Council (ERC) Starting Grant (ERC-2014-STG-639526, NANOGEN). S.K.-N. and Q.J. S.K.-N. and Q.J. acknowledge support from the EPSRC Centre of Advanced Materials for Integrated Energy Systems "CAM-IES" (grant EP/P007767/1). We thank Henry Lloyd-Laney and Ezra Kitson for helpful discussions in statistical analysis. We thank Professor. Nicolas Plumeré and Dr. Huaiguang Li for helpful discussions in electrochemistry. We thank Professor. Paulo J. Bártolo and Dr. Fengyuan Liu for helpful discussions in 3D bioprinting.

\section{Author Contributions}

XC performed all experiments; JZ and XC wrote the manuscript and conceived the project. JML performed the statistical analysis. LTW, JML and CH contributed to the biological work, QJ and SNK contributed to the aerosol jet printing method, LS and SV contributed to the light measurements. All authors contributed to discussions and interpretations of the results and have given approval to the final version of the manuscript.

\section{Conflicts of interest}

There are no conflicts to declare. 


\section{References}

1 Kornienko, N., Zhang, J., Sakimoto, K., Yang, P. \& Reisner, E. Interfacing nature's catalytic machinery with synthetic materials for semi-artificial photosynthesis. Nature Nanotechnology 13, 890-899, doi:10.1038/s41565-018-0251-7 (2018). Zhang, J. \& Reisner, E. Advancing photosystem II photoelectrochemistry for semi-artificial photosynthesis. Nature Reviews Chemistry 4, 6-21, doi:10.1038/s41570-019-0149-4 (2020). King, P. W. Semi-synthetic strategy. Nature Energy 3, 921-922 (2018).

4 Léger, C. \& Bertrand, P. Direct electrochemistry of redox enzymes as a tool for mechanistic studies. Chemical Reviews 108, 2379-2438 (2008).

5 Saar, K. L. et al. Enhancing power density of biophotovoltaics by decoupling storage and power delivery. Nature energy 3, 75-81 (2018).

6 Wey, L. T. et al. The development of biophotovoltaic systems for power generation and biological analysis. ChemElectroChem 6, 5375-5386 (2019).

7 Zhang, J. Z. et al. Photoelectrochemistry of photosystem II in vitro vs in vivo. Journal of the American Chemical Society 140, 6-9 (2018).

8 McCormick, A. J. et al. Biophotovoltaics: oxygenic photosynthetic organisms in the world of bioelectrochemical systems. Energy \& Environmental Science 8, 1092-1109 (2015).

9 Bradley, R. W., Bombelli, P., Lea-Smith, D. J. \& Howe, C. J. Terminal oxidase mutants of the cyanobacterium Synechocystis sp. PCC 6803 show increased electrogenic activity in biological photo-voltaic systems. Physical Chemistry Chemical Physics 15, 13611-13618 (2013).

10 Sekar, N., Jain, R., Yan, Y. \& Ramasamy, R. P. Enhanced photo-bioelectrochemical energy conversion by genetically engineered cyanobacteria. Biotechnology and bioengineering 113, 675-679 (2016).

11 Liu, C., Dasgupta, N. P. \& Yang, P. Semiconductor nanowires for artificial photosynthesis. Chemistry of Materials 26, 415-422 (2014).

12 Mersch, D. et al. Wiring of photosystem II to hydrogenase for photoelectrochemical water splitting. Journal of the American Chemical Society 137, 8541-8549 (2015).

13 Fang, X., Kalathil, S., Divitini, G., Wang, Q. \& Reisner, E. A three-dimensional hybrid electrode with electroactive microbes for efficient electrogenesis and chemical synthesis. Proceedings of the National Academy of Sciences 117, 5074-5080 (2020).

14 Sokol, K. P. et al. Rational wiring of photosystem II to hierarchical indium tin oxide electrodes using redox polymers. Energy \& Environmental Science 9, 3698-3709 (2016).

15 Wenzel, T., Härtter, D., Bombelli, P., Howe, C. J. \& Steiner, U. Porous translucent electrodes enhance current generation from photosynthetic biofilms. Nature communications 9, 1-9 (2018).

16 Sturmberg, B. C. et al. Modal analysis of enhanced absorption in silicon nanowire arrays. Optics Express 19, A1067-A1081 (2011).

17 Ali, M. et al. Nanostructured photoelectrochemical solar cell for nitrogen reduction using plasmon-enhanced black silicon. Nature communications 7, 1-5 (2016).

18 Torimura, M., Miki, A., Wadano, A., Kano, K. \& Ikeda, T. Electrochemical investigation of cyanobacteria Synechococcus sp. PCC7942-catalyzed photoreduction of exogenous quinones and photoelectrochemical oxidation of water. Journal of Electroanalytical Chemistry 496, 2128 (2001).

19 Reggente, M., Politi, S., Antonucci, A., Tamburri, E. \& Boghossian, A. A. Design of optimized PEDOT-based electrodes for enhancing performance of living photovoltaics based on phototropic bacteria. Advanced Materials Technologies 5, 1900931 (2020).

20 Cereda, A. et al. A bioelectrochemical approach to characterize extracellular electron transfer by Synechocystis sp. PCC6803. PLoS One 9, e91484 (2014). 
21 Zeng, Y. et al. Photoactive conjugated polymer-based hybrid biosystems for enhancing cyanobacterial photosynthesis and regulating redox state of protein. Advanced Functional Materials 31, 2007814 (2021).

22 Wang, K., Chang, Y.-H., Zhang, C. \& Wang, B. Conductive-on-demand: Tailorable polyimide/carbon nanotube nanocomposite thin film by dual-material aerosol jet printing. Carbon 98, 397-403 (2016).

23 Saleh, M. S., Li, J., Park, J. \& Panat, R. 3D printed hierarchically-porous microlattice electrode materials for exceptionally high specific capacity and areal capacity lithium ion batteries. Additive Manufacturing 23, 70-78 (2018).

24 Saleh, M. S., Hu, C. \& Panat, R. Three-dimensional microarchitected materials and devices using nanoparticle assembly by pointwise spatial printing. Science advances 3, e1601986 (2017).

25 Ćatić, N. et al. Aerosol-jet printing facilitates the rapid prototyping of microfluidic devices with versatile geometries and precise channel functionalization. Applied Materials Today 19, 100618 (2020).

26 Jabari, E. \& Toyserkani, E. Micro-scale aerosol-jet printing of graphene interconnects. Carbon 91, 321-329 (2015).

27 Sukeshini, A. M. et al. Aerosol jet printing and microstructure of SOFC electrolyte and cathode layers. ECS Transactions 35, 2151 (2011).

28 Kalio, A. et al. Development of lead-free silver ink for front contact metallization. Solar energy materials and solar cells 106, 51-54 (2012).

29 Lu, S. et al. Flexible, print-in-place 1D-2D thin-film transistors using aerosol jet printing. ACS nano 13, 11263-11272 (2019).

30 Hong, K. et al. Aerosol jet printed, Sub-2 V complementary circuits constructed from P- and Ntype electrolyte gated transistors. Advanced Materials 26, 7032-7037 (2014).

31 Martinez-Acosta, A., Tafoya, R. R., Quinones, S. A. \& Secor, E. B. Modular motion control software development to support a versatile, low-cost aerosol jet platform for printed electronics. Additive Manufacturing, 101932 (2021).

32 Tafoya, R. R. et al. Real-time optical process monitoring for structure and property control of aerosol jet printed functional materials. Advanced Materials Technologies 5, 2000781 (2020).

33 Mahajan, A., Frisbie, C. D. \& Francis, L. F. Optimization of aerosol jet printing for highresolution, high-aspect ratio silver lines. ACS applied materials \& interfaces 5, 4856-4864 (2013).

34 Kopola, P. et al. Aerosol jet printed grid for ITO-free inverted organic solar cells. Solar energy materials and solar cells 107, 252-258 (2012).

35 Williams, B. A. et al. Formation of copper zinc tin sulfide thin films from colloidal nanocrystal dispersions via aerosol-jet printing and compaction. ACS applied materials \& interfaces 7 , 11526-11535 (2015).

36 Yang, C., Zhou, E., Miyanishi, S., Hashimoto, K. \& Tajima, K. Preparation of active layers in polymer solar cells by aerosol jet printing. ACS applied materials \& interfaces 3, 4053-4058 (2011).

37 Fang, X. et al. Structure-activity relationships of hierarchical three-dimensional electrodes with photosystem II for semiartificial photosynthesis. Nano letters 19, 1844-1850 (2019).

38 Lin, J.-T. et al. A high-efficiency HIT solar cell with pillar texturing. IEEE Journal of Photovoltaics 8, 669-675 (2018).

39 Saini, D. K., Pabbi, S. \& Shukla, P. Cyanobacterial pigments: Perspectives and biotechnological approaches. Food and chemical toxicology 120, 616-624 (2018).

40 Clifford, E. R. et al. Phenazines as model low-midpoint potential electron shuttles for photosynthetic bioelectrochemical systems. Chemical Science 12, 3328-3338 (2021).

41 Beauzamy, L. et al. Mediator-microorganism interaction in microbial solar cell: a fluo electrochemical insight. Analytical chemistry 92, 7532-7539 (2020). 
42 Stanier, R., Kunisawa, R., Mandel, M. \& Cohen-Bazire, G. Purification and properties of unicellular blue-green algae (order Chroococcales). Bacteriological reviews 35, 171 (1971).

43 Lea-Smith, D. J. et al. Thylakoid terminal oxidases are essential for the cyanobacterium Synechocystis sp. PCC 6803 to survive rapidly changing light intensities. Plant physiology 162, 484-495 (2013).

44 Team, R. C. R: A language and environment for statistical computing. (2013).

45 Wei, T. \& Simko, V. package "corrplot": Visualization of a correlation matrix (Version 0.84) Statistician 56, e24 (2017). 